\title{
Editorial
}

\section{Screening for Bipolar Disorder: Confusion between Case-Finding and Screening}

\author{
Mark Zimmerman \\ Department of Psychiatry and Human Behavior, Brown Medical School, and Department of Psychiatry, Rhode Island \\ Hospital, Providence, R.I., USA
}

\section{Introduction}

Bipolar disorder is a serious illness resulting in significant psychosocial morbidity and excess mortality [1]. The prevalence of bipolar disorder is relatively high, especially when it is defined to include milder variants such as bipolar II disorder and bipolar disorder not otherwise specified [2].

Despite its clinical and public health significance, studies of psychiatric and primary care patients have found that bipolar disorder is often missed [3-5]. The underdiagnosis of bipolar disorder in depressed patients has potential treatment and clinical implications such as the underprescription of mood-stabilizing medications, an increased risk of rapid cycling due to possible overprescription of antidepressant medications and increased costs of care [3,6-8].

Several causes of bipolar disorder underdiagnosis have been posited. Patients with bipolar disorder are more likely to experience symptoms of depression and anxiety, and present for treatment of these symptoms rather than symptoms of mania or hypomania $[9,10]$. Because patients with bipolar disorder usually do not present in the manic or hypomanic phase of the illness, to correctly diagnose bipolar disorder, the presence of a prior history of hypomanic or manic episodes must be elicited. Recommendations for improving the detection of bipolar disorder include careful clinical evaluations, inquiring about a history of mania and hypomania, and the use of screening questionnaires [11-13].

Many scales have been developed to assess manic/hypomanic symptoms. Some of these instruments are crosssectional measures of symptom severity, and, prior to DSM-5, would not have been appropriate as diagnostic aids in currently depressed patients. The reason for the caveat regarding DSM-5 is because DSM-5 includes a mixed feature specifier for depressed patients which these scales might be useful in identifying. In the past few years, 4 self-report questionnaires have been developed to screen for bipolar disorder [14-17]. That is, they assess a lifetime history of manic/hypomanic symptoms. The purpose of the present article is twofold. First, I briefly describe the structure and content of these 4 scales and summarize the initial studies establishing the cutoff score derived by the scale developers that was recommended to screen for bipolar disorder. Second, I discuss the distinction between case-finding and screening, how this distinction is reflected in the derivation of a measure's cutoff score, and examine how this applies to the 4 bipolar disorder screening instruments.

\section{KARGER}

E-Mail karger@karger.com www.karger.com/pps
(C) 2014 S. Karger AG, Basel

0033-3190/14/0835-0259\$39.50/0
Mark Zimmerman, MD

Department of Psychiatry

Rhode Island Hospital, 146 W River Street

Providence, RI 02904 (USA)

E-Mail mzimmerman@lifespan.org 


\section{Description of Four Screening Scales for Bipolar Disorder}

\section{The Hypomanic Checklist}

The goal of the Hypomanic Checklist (HCL-32) was to improve the recognition of hypomanic features in depressed patients and thereby enhance recognition of bipolar II disorder and other bipolar spectrum disorders [16]. The introduction to the HCL-32 notes: 'At different times in their life everyone experiences changes or swings in energy, activity and mood ("highs and lows" or "ups and downs"). The aim of this questionnaire is to assess the characteristics of the "high" periods.' The respondent is then instructed to think of a 'period when you were in a "high" state' and answer 32 yes-no questions about their mood and behavior during that time. Each no response is scored 0 and each yes response is scored 1 , and the total HCL-32 score is obtained by summing the number of yes responses. Thus, the range of scores spans from 0 to 32 . There are some additional items to the scale assessing other clinically important variables such as functional impairment and symptom duration, but these do not contribute to the total score and do not contribute to determining whether the respondent screens positive. The scale is reprinted in the published article.

The initial study of the HCL-32 was of 446 Italian and Swedish patients (all but 7 were outpatients) with major depressive disorder or bipolar disorder. The authors recommended a cutoff score of 14 because it yielded the best combination of sensitivity (80\%) and specificity (51\%) in distinguishing bipolar disorder from major depressive disorder. The authors did not present the sensitivity and specificity values for other cutoff scores.

\section{Bipolar Spectrum Diagnostic Scale}

The authors of the Bipolar Spectrum Diagnostic Scale (BSDS) had conducted a previous study of the Mood Disorders Questionnaire and concluded that the scale had good sensitivity for bipolar I disorder (70\%) but a less impressive sensitivity for bipolar II disorder and bipolar disorder not otherwise specified [18]. The goal of the BSDS was to improve the recognition of milder, subthreshold, forms of the bipolar spectrum and thus be effective across the entire bipolar spectrum severity range [19]. The scale consists of two parts. The first part is a paragraph containing 19 statements describing many of the symptoms of bipolar disorder, with each sentence followed by an underlined space for respondents to place a checkmark if they believe that the statement applies to them. In scoring the scale, each item checked is counted as 1 point. The second part of the BSDS is a single multiple-choice question asking respondents how well the paragraph describes them [very well, or almost perfectly (6 points); fairly well (4 points); to some degree, but not in most respects ( 2 points); not really at all (0 points)]. Thus, the range of scores spans from 0 to 25 . The instructions of the BSDS ask patients to read through the entire paragraph before checking off which symptom statements apply to them. After reading the paragraph, patients are asked to complete the multiple-choice question, and then to go back and put a check mark after each statement that 'definitely describes you'. A copy of the BSDS is reprinted in the article by Ghaemi et al. [19].

The initial study of the BSDS was of 71 patients with major depressive disorder or bipolar disorder. A priori the authors suggested that a cutoff of 12 represented a positive screen. At this cutoff the sensitivity of the BSDS was $76 \%$, and its specificity was $85 \%$. They examined the performance of the scale at other cutoff points and recommended a cutoff score of 13 because it yielded the best combination of sensitivity (75\%) and specificity (93\%) in distinguishing bipolar disorder from major depressive disorder. The authors presented the sensitivity and specificity values for cutoff scores of 10 through 14 in a figure.

\section{Mood Swings Questionnaire/Survey}

In the initial publication this scale was referred to as the Mood Swings Survey [20], but more recently it has been called the Mood Swings Questionnaire (MSQ), and I will therefore use this name $[21,22]$.

The goal of the MSQ was to improve the recognition of bipolar II disorder in depressed patients and to better distinguish bipolar I and bipolar II disorder. As described in early publications [21], the MSQ begins with a 2-part screening question: 'Do you ever have mood swings, and as part of such swings, have times when (i) your mood is higher than your usual sense of happiness ("a high") and (ii) you feel quite "wired", "energized", "elevated", "expansive", and possibly "irritable"?' If the respondent answers no to this question, the remainder of the scale is not completed. (In more recent descriptions, including the version available on the Black Dog Institute website, this complex question has been simplified. However, the approach remains the same insofar as the subsequent questions are not completed if the screening question is answered in the negative.) This question is followed by 46 questions rated on a 3-point scale [no more than usual (scored 0); somewhat more than usual (scored 1); much more than usual (scored 2)]. A 27-item version of the scale has also been developed. The range of scores spans 
from 0 to 92 ( 0 to 54 for the shorter version). The briefer 27 -item scale is reprinted on the Black Dog Institute website.

The initial study of the MSQ-46 was of 157 depressed outpatients. The authors recommended a cutoff score of 36 as optimal in distinguishing bipolar disorder and major depressive disorder. At this cutoff the scale achieved a sensitivity of $84 \%$ and a specificity of $93 \%$. For the shortened 27 -item version, the optimal cutoff of 22 resulted in a sensitivity of $81 \%$ and a specificity of $98 \%$. The authors did not present the sensitivity and specificity values for other cutoff scores.

\section{Mood Disorders Questionnaire}

The Mood Disorders Questionnaire screens for a lifetime history of mania or hypomania with 13 yes/no symptom questions reflecting the DSM-IV inclusion criteria [15]. Total scores are calculated by summing the 13 items that are answered yes. The symptom questions are followed by a single yes/no question about whether the symptoms clustered during the same period of time. The respondent is instructed to answer this question only if more than 1 symptom was checked off. The final question evaluates the level of impairment resulting from the symptoms. This item is rated on a 4-point scale (no problem, minor problem, moderate problem, serious problem).

The initial study of the MDQ was of 198 outpatients treated in clinics specializing in the treatment of mood disorders, especially bipolar disorder. The authors recommended a cutoff score of 7 symptoms that co-occurred and caused moderate or serious problems because it yielded the best combination of sensitivity (73\%) and specificity (90\%) in distinguishing bipolar disorder from major depressive disorder. The authors presented a graph of the sensitivity and specificity of the scale for all symptom cutoff scores.

\section{Screening or Case-Finding?}

The threshold chosen to identify cases on a symptom questionnaire with a continuous score distribution is based on the intended use of the scale. If the goal is to identify a relatively homogeneous group of individuals who are highly likely to have the index disorder, a high threshold will be chosen in order to increase the specificity of the scale and reduce the number of false-positive results in the group scoring above the threshold. To achieve this, high sensitivity is sacrificed. On the other hand, if the purpose is to use the scale as screening measure, as purportedly each of these tests was intended to be, then a broad net needs to be cast and the threshold set low in order to enhance the sensitivity of the measure. It is important for a screening scale to have high sensitivity because the more time-intensive follow-up diagnostic inquiry will presumably only occur in patients who are positive on the initial screen. Clinicians who rely on the screening scale as the first stage of a 2-stage diagnostic assessment, with the second stage being the more indepth diagnostic interview, will miss the diagnosis in patients who are false-negative on the instrument.

In developing each of these scales, the 4 research groups recommended a cutoff score that provided the best balance between sensitivity and specificity thereby optimizing agreement between the test and the diagnostic standard. While there is nothing inherently wrong with this method of selecting a cutoff score, it is at odds with the stated intended use of the scale as a screening instrument. In the initial validation study of 3 of the 4 scales, the sensitivity of the measure at the recommended cutoff score was lower than its specificity.

None of the scale developers discussed how their test should be used in real-world clinical practice. Consistently with the concept of screening, the scales should be administered prior to the clinical interview, with the clinician following up positive screens. Did the authors envision an alternative manner of using the scale? Did the authors expect clinicians to inquire about a history of manic and hypomanic episodes for patients who screened negative on the scale? If not, then scale sensitivity is of critical importance. If so, then what is the purpose of the scale? Do the authors of these scales believe that clinicians do not know what questions to ask to assess prior manic/ hypomanic episodes and therefore these scales are needed because the scales ask the right questions that clinicians do not? Presumably the scale developers expected clinicians to follow up positive screens with a diagnostic interview because they did not suggest that these self-administered scales are definitive diagnostic tests. Moreover, from a feasibility of clinical use perspective, none of the scale developers attempted to develop as brief a measure as possible, which would be desirable in a screening instrument that could be used alongside measures that screen for other disorders that are important to recognize in depressed patients. A screening measure does not need to be comprehensive and cover all of the criteria of mania/ hypomania, but instead needs to include a sufficient number of items to achieve a high sensitivity and acceptable specificity. 
None of the scale developer groups discussed the possibility of false-positive diagnoses associated with falsepositive screening test results which would occur because a clinician was overly influenced by the results of the screening test. False-positive cases are at risk of overtreatment with mood stabilizers. For other medical disorders, screening recommendations typically include a discussion of both potential benefits and harms. There are several examples in the research literature in which the results of bipolar disorder screening tests were misinterpreted as suggestive of actual caseness rather than screen positives [23]. If researchers (as well as the reviewers of these articles published in peer review journals) misinterpret the results of these tests, are clinicians likely to do so as well?
Recommendations to use screening scales for bipolar disorder in clinical practice are common [24-27]. While the underdiagnosis of bipolar disorder is a problem in clinical practice, a balanced discussion of the potential risks and benefits of screening has been lacking. It is important for the developers of screening scales, and investigators of the performance of these measures, to discuss their rationale for selecting cutoff scores that maximize agreement with a diagnostic standard rather than selecting a cutoff score that favors sensitivity over specificity.

\section{Disclosure Statement}

The author has no conflict of interest to declare.

\section{References}

1 Murray C, Lopez A: Alternative projections of mortality and disability by cause $1990-2020$ : global burden of disease study. Lancet 1997; 349:1498-1504.

2 Merikangas KR, Akiskal HS, Angst J, Greenberg PE, Hirschfeld RM, Petukhova M, Kessler RC: Lifetime and 12-month prevalence of bipolar spectrum disorder in the National Comorbidity Survey replication. Arch Gen Psychiatry 2007;64:543-552.

-3 Ghaemi SN, Boiman EE, Goodwin FK: Diagnosing bipolar disorder and the effect of antidepressants: a naturalistic study. J Clin Psychiatry 2000;61:804-808.

4 Ghaemi SN, Ko JY, Goodwin FK: 'Cade's disease' and beyond: misdiagnosis, antidepressant use, and a proposed definition for bipolar spectrum disorder. Can J Psychiatry 2002;47: 125-134.

5 Hantouche EG, Akiskal HS, Lancrenon S, Allilaire JF, Sechter D, Azorin JM, Bourgeois M, Fraud JP, Chatenet-Duchene L: Systematic clinical methodology for validating bipolar-II disorder: data in mid-stream from a French national multi-site study (EPIDEP). J Affect Disord 1998;50:163-173.

6 6 Birnbaum HG, Shi L, Dial E, Oster EF, Greenberg PE, Mallett DA: Economic consequences of not recognizing bipolar disorder patients: a cross-sectional descriptive analysis. J Clin Psychiatry 2003;64:1201-1209.

7 Shi L, Thiebaud P, McCombs JS: The impact of unrecognized bipolar disorders for patients treated for depression with antidepressants in the fee-for-services California Medicaid (Medi-Cal) program. J Affect Disord 2004;82: 373-383.

8 Matza LS, Rajagopalan KS, Thompson CL, de Lissovoy G: Misdiagnosed patients with bipolar disorder: comorbidities, treatment patterns, and direct treatment costs. J Clin Psychiatry 2005;66:1432-1440.
9 Judd L, Akiskal H, Schettler P, Endicott J, Maser J, Solomon D, Leon A, Rice J, Keller M: The long-term natural history of the weekly symptomatic status of bipolar I disorder. Arch Gen Psychiatry 2002;59:530-537.

10 Judd L, Akiskal H, Schettler P, Coryell W, Endicott J, Maser J, Solomon D, Leon A, Keller $\mathrm{M}$ : A prospective investigation of the natural history of the long-term weekly symptomatic status of bipolar II disorder. Arch Gen Psychiatry 2003;60:261-269.

11 Hirschfeld RM, Vornik LA: Recognition and diagnosis of bipolar disorder. J Clin Psychiatry 2004;65(suppl 15):5-9.

12 Yatham LN: Diagnosis and management of patients with bipolar II disorder. J Clin Psychiatry 2005;66(suppl 1):13-17.

13 Bowden CL: Strategies to reduce misdiagnosis of bipolar depression. Psychiatr Serv 2001;52: 51-55.

14 Depue RA, Krauss S, Spoont MR, Arbisi P: General behavior inventory identification of unipolar and bipolar affective conditions in a nonclinical university population. J Abnorm Psychol 1989;98:117-126.

15 Hirschfeld R, Williams J, Spitzer R, Calabrese J, Flynn L, Keck P Jr, Lewis L, McElroy S, Post R, Rapport D, Russell J, Sachs G, Zajecka J: Development and validation of a screening instrument for bipolar spectrum disorder: the Mood Disorder Questionnaire. Am J Psychiatry 2000;157:1873-1875.

16 Angst J, Adolfsson R, Benazzi F, Gamma A, Hantouche E, Meyer TD, Skeppar P, Vieta E, Scott J: The HCL-32: towards a self-assessment tool for hypomanic symptoms in outpatients. J Affect Disord 2005;88:217-233.

17 Bowden CL, Singh V, Thompson P, Gonzalez JM, Katz MM, Dahl M, Prihoda TJ, Chang X: Development of the bipolar inventory of symptoms scale. Acta Psychiatr Scand 2007; 116:189-194.
18 Miller CJ, Klugman J, Berv DA, Rosenquist KJ, Ghaemi SN: Sensitivity and specificity of the Mood Disorder Questionnaire for detecting bipolar disorder. J Affect Disord 2004;81: 167-171.

19 Ghaemi SN, Miller CJ, Berv DA, Klugman J, Rosenquist KJ, Pies RW: Sensitivity and specificity of a new bipolar spectrum diagnostic scale. J Affect Disord 2005;84:273-277.

20 Parker G, Hadzi-Pavlovic D, Tully L: Distinguishing bipolar and unipolar disorders: an isomer model. J Affect Disord 2006;96:67-73.

21 Parker G, Fletcher K, Barrett M, Synnott H, Breakspear M, Hyett M, Hadzi-Pavlovic D: Screening for bipolar disorder: the utility and comparative properties of the MSS and MDQ measures. J Affect Disord 2008;109:83-89.

22 Parker G, Graham R, Hadzi-Pavlovic D, Fletcher K, Hong M, Futeran S: Further examination of the utility and comparative properties of the MSQ and MDQ bipolar screening measures. J Affect Disord 2012;138: 104-109.

23 Zimmerman M: Misuse of the Mood Disorders Questionnaire as a case-finding measure and a critique of the concept of using a screening scale for bipolar disorder in psychiatric practice. Bipolar Disord 2012;14:127-134.

24 Chessick CA, Dimidjian S: Screening for bipolar disorder during pregnancy and the postpartum period. Arch Women's Mental Health 2010;13:233-248.

25 Loganathan M, Lohano K, Roberts RJ, Gao Y, El-Mallakh RS: When to suspect bipolar disorder. J Fam Pract 2010;59:682-688.

26 Frye MA: Clinical practice. Bipolar disorder - a focus on depression. N Engl J Med 2011; 364:51-59.

27 Anderson IM, Haddad PM, Scott J: Bipolar disorder. BMJ 2012;345:e8508. 